CAHIERS DE

NARRATOLOGIE

\section{Cahiers de Narratologie}

Analyse et théorie narratives

14 | 2008

Prose d'idées : formes et savoirs

\title{
Théâtralité et narration : Le Bonheur dans le crime de Barbey d'Aurevilly
}

\section{Kris Vassilev}

\section{(2) OpenEdition}

Journals

Édition électronique

URL : http://journals.openedition.org/narratologie/594

DOI : $10.4000 /$ narratologie.594

ISSN : 1765-307X

Éditeur

LIRCES

Référence électronique

Kris Vassilev, "Théâtralité et narration : Le Bonheur dans le crime de Barbey d'Aurevilly », Cahiers de Narratologie [En ligne], 14 | 2008, mis en ligne le 06 mars 2008, consulté le 19 avril 2019. URL : http:// journals.openedition.org/narratologie/594; DOI : 10.4000/narratologie.594

Ce document a été généré automatiquement le 19 avril 2019

\section{(c) $($ i) $(9)$}

Cahiers de Narratologie - Analyse et théorie narratives est mis à disposition selon les termes de la licence Creative Commons Attribution - Pas d'Utilisation Commerciale - Pas de Modification 4.0 International. 


\title{
Théâtralité et narration : Le Bonheur dans le crime de Barbey d'Aurevilly
}

\author{
Kris Vassilev
}

1 À comparer la préface des Diaboliques, telle qu'elle apparait dans la première publication de l'ouvrage chez l'éditeur Dentu en 1874, à son esquisse datée de 1870 que l'on retrouve dans les cahiers de notes de Barbey, Disjecta Membra, on constate d'emblée une différence de taille. Les retouches stylistiques mises à part, la version définitive de cette préface diffère de sa première mouture en ce qu'elle contient une espèce de "défense et illustration » de la valeur morale des Diaboliques. Le rajout auquel procède Barbey a bien entendu été remarqué et commenté par la critique. Le but de cette rectification tardive, tel est l'avis commun, serait de dissuader d'éventuelles objections portant sur l'aspect subversif de l'œuvre; c'est « un paratonnerre contre un scandale que Barbey voit venir », écrit par exemple Michel Crouzet à ce propos. ${ }^{1}$ Cette observation est d'autant plus irréfutable que peu après la parution des Diaboliques, comme on sait, leur auteur fait l'objet d'un procès pour outrage à la morale publique.

2 Jacques Petit souligne avec justesse que la refonte du projet de préface relève surtout d'une « justification morale de l'ouvrage ; brève, mais essentielle ». ${ }^{2}$ Crouzet reprendra presque la même formule pour rappeler que "seule la seconde (version) présente une justification morale et religieuse du livre $»^{3}$ Les exégètes des Diaboliques, semblent s'être accordés pour conférer une même et seule signification à cet "adoucissement", l'inscrivant sur le compte d'une stratégie de prévention. Or, à bien examiner les termes que Barbey a choisis pour remanier son texte, et plus particulièrement ceux qu'il a voulu rendre ostensibles en les italicisant, on entrevoit une dimension autre de cette modification; dimension certes inextricablement liée à la valeur justificatrice, mais néanmoins bien distincte.

3 Quel que soit le sujet traité par les peintres de talent, explique Barbey, « leur peinture est toujours assez morale, quand elle est tragique et qu'elle donne l'horreur des choses qu'elle retrace. " Esthétique et éthique, que le réalisme et le symbolisme ambiants tendent à séparer, font ici cause commune. Afin de mettre en valeur l'exemplarité morale de son 
œuvre, Barbey ira jusqu'à adopter le langage du prédicateur: «Quand on aura lu ces Diaboliques, je ne crois pas qu'il y ait une seule personne en disposition à les recommencer en fait, et toute la moralité d'un livre est là... » (1290-1) La visée ultime des Diaboliques, à en croire leur auteur, serait de donner une leçon, d'instruire. "Morale», «tragique ", " retracer ", « horreur ", « tragédie ", voici les termes que Barbey veut faire retenir à son public. Cette justification, qui inaugure l'œuvre autant qu'elle programme sa lecture, n'est pas étrangère aux principes gouvernant la tragédie classique. L'auteur des Diaboliques semble vouloir instaurer un parallèle entre l'effet que son ouvrage se propose d'obtenir, par l'intermédiaire de la terreur suscitée, et la fonction primordiale du genre tragique, à savoir l'épuration des âmes et l'éradication des mauvaises passions. Du reste, c'est autour du principe purificateur de l'œuvre littéraire que Barbey organisera sa plaidoirie devant le tribunal. « Le but de mon œuvre a été de moraliser mes semblables en leur donnant l'horreur du vice. L'immoralité, quand elle est cauteleuse, pénètre dans les masses, elles la rejettent au contraire, quand elle est terrible.»C'est la pensée de " moraliser par la terreur » qui se tient à la source des Diaboliques, insiste encore Barbey avant de synthétiser son propos en guise de conclusion: les nouvelles sont conçues «dans le seul but d'épurer et de fortifier. $»^{4}$

4 Mais si ce véritable arsenal d'expressions aux accents explicitement moralisateurs nous autorise à évoquer les enjeux éthiques de la tragédie, l'écriture des Diaboliques, elle, donne à voir un rapport plus général entre théâtre et récit, en raison de l'importance décisive conférée, au sein de la narration, au concept de spectacle. La présence de la thématique théâtrale, plus ou moins clairement discernable dans chaque nouvelle du recueil, circule d'un texte à l'autre et confirme à son tour la forte cohésion structurelle et sémantique de l'œuvre. Un critique comme Julien Gracq - le seul à notre connaissance à avoir insisté de manière systématique sur ce phénomène - voit la théâtralité des Diaboliques se manifester essentiellement sur deux plans; celui d'abord de l'interaction conteur/auditeur imprégnée des marques de l'oralité, grâce à «l'échange continuel de signes, la mimique théâtrale et expressive de la présence $»^{5}$, mais aussi à l'aide de "cette constante vérification du contact »; et puis celui de l'émotion que l'auteur cherche à communiquer à son lecteur, «amplifiée, réfractée, agrandie, jouée comme au théâtre par les mouvements communicatifs d'un auditoire supposé sous le charme et rendu à chaque instant idéalement présent par l'art du conteur ${ }^{6}{ }^{6}$

5 Une analyse d'ensemble des Diaboliques, envisagées sous l'angle de la théâtralité, exigerait une étude plus ample, opérant un va-et-vient constant entre les différentes nouvelles. D'où notre choix de consacrer la présente analyse à un seul récit, Le Bonheur dans le crime. Ce choix peut cependant étonner: certains textes des Diaboliques semblent a priori se prêter mieux à une analyse de la dimension théatrale. On peut penser par exemple au Plus bel amour de Don Juan, où la référence au théâtre s'impose dès le titre de la nouvelle, se maintient dans la distribution du récit en chapitres (conforme au découpage en cinq actes de la tragédie classique), et résonne jusque dans le sobriquet, "la petite masque", attribué au personnage de la jeune fille $e^{7}$ ou encore au Dessous de cartes d'une partie de whist, où l'auteur s'essaie à une autopsie du "théâtre social » dans l'espace du salon aristocratique, de même qu'il attire l'intérêt sur les effets de la réception chez un public à l'intérieur de la fiction. Ce qui rend cependant Le Bonheur dans le crime particulièrement fascinant dans l'optique de la théâtralité, c'est que l'intrigue repose ici entièrement sur le jeu de théâtre auquel s'adonnent les personnages et sans lequel, au fond, le récit n'existerait pas. Il semble donc pour le moins logique que l'observation et le plaisir qu'elle 
procure dominent la narration qui y puise son matériau et y trouve sa légitimité. De ce fait, le lecteur du Bonheur lui-même se retrouve placé dans la position ambiguë de spectateur. C'est de cette imbrication du narratif et du théatral, de cette collaboration entre récit et spectacle, entre « raconter» et «présenter » que l'on cherche à rendre compte ici. ${ }^{8}$

6 Il s'agira alors de vérifier si cette théâtralité est en phase avec le régime cathartique de la tragédie classique, dont l'écriture des Diaboliques (à prendre à la lettre sa seconde préface) semble se réclamer lorsqu'elle invoque une justification liée à l'effet qu'elle est censée produire. En d'autres mots, la question se pose de savoir si la théâtralisation de la nouvelle, présentée comme inhérente à l'intention d'édification, parvient à susciter le fonctionnement éthico-esthétique du système tragique, ou si elle l'enraye au contraire.

7 Il suffit d'envisager sa réception et «les horizons d'attente » qui s'y rattachent pour constater que toute nouvelle présente des affinités avec le genre dramatique. Comme chez toutes les formes brèves, le facteur temporel joue ici un rôle déterminant tant sur le plan de la composition que sur celui de la perception du texte. Le temps nécessaire à la lecture de la nouvelle se réduit généralement à la durée d'un spectacle. De toute évidence, dans les deux cas, la contrainte temporelle correspond à l'exigence d'unité dramatique: concentration de l'intrigue, restriction du nombre de personnages, rétrécissement de l'espace, etc. La réduction des composantes et la concision dramatique, explique Daniel Grojnowski, donnent au lecteur de la nouvelle l'impression d'une emprise sur l'ensemble narratif et " permet une représentation mentale complète. "

La forme condensée de la nouvelle, qui garantit sa consommation ininterrompue, constitue ce que les théoriciens du récit court, depuis Poe et Baudelaire jusqu'à Borges, en passant par O'Connor, considèrent comme étant son principe de base: la possibilité d'appréhension globale du contenu narratif. «Chaque terme trouve sa résonance du fait que le lecteur garde en mémoire un grand nombre de données. Il jouit d'une perception panoramique, ni le détail ni la totalité ne lui échappent. Ce point de vue d'observateur lui est source de plaisir car il se trouve en face d'un tableau synoptique. $»^{10}$ Voilà la lecture de la nouvelle définie comme l'expérience d'une observation in extenso et le lecteur lui-même comme un spectateur. Dans la perspective qui est la nôtre, cette formulation est particulièrement significative ; elle articule la lecture du récit bref à la possibilité de sa visualisation et par là même autorise à dépasser une analogie depuis longtemps instituée avec la peinture, pour suggérer des rapports plus complexes :

À la métaphore picturale on peut préférer la référence théâtrale, telle du moins que la prône l'esthétique classique : unité de lieu, de temps et d'action, avec un nombre limité de protagonistes. [...] Bien que de tels rapprochements ne rendent pas compte de la nouvelle en tant que récit, ils la caractérisent dans sa morphologie et ils explicitent le plaisir qu'éprouve le lecteur à découvrir une aventure qu'il maitrise (qu'il croit maîtriser...) de son commencement jusqu'à son terme. ${ }^{11}$

9 Ainsi, le caractère théâtral du récit de nouvelle se fait doublement sentir : dans la mise en place des éléments structurels (la morphologie), aussi bien que dans le registre de la perception (l'effet de totalité). Dans la mesure où le Bonheur construit un espace narratif où l'acte de raconter est entièrement soumis à l'acte de voir, tandis que les personnages sont présentés comme des acteurs, interprétant des rôles et se donnant en spectacle, ${ }^{12} \mathrm{il}$ s'agira pour nous d'examiner le rapport qu'entretiennent narration et théâtre et d'articuler les implications qui en résultent. 
10 On connaît les grandes lignes de la nouvelle. Un narrateur, qui n'est pas nommé, raconte à « Madame » l'histoire que lui a contée le docteur Tory, au cours d'une promenade dans le Jardin des Plantes. On y rencontre un couple fascinant, tant par l'apparence que par le comportement. Il s'agit du comte Serlon de Savigny et de sa seconde femme, l'ancienne experte en escrime, Hauteclaire. S'arrêtant devant la cage d'une panthère, celle-ci provoque la bête sauvage en la frappant sur le museau avec son gant. La panthère réagit d'un coup de mâchoire foudroyant, mais ses dents ne se referment que sur l'étoffe du gant, la main s'étant retirée in extremis. Cet incident, qui produit une émotion partagée sur le groupe d'observateurs, déclenche le récit du docteur. Le métier que celui-ci exerçait à l'époque des événements qu'il évoque, lui permettait de pénétrer la vie intime de ses patients. Or, la feue comtesse Savigny, atteinte d'une maladie chronique, faisait partie de sa clientèle. Lors de ses visites au château des Savigny, le médecin reconnaît, dans Eulalie, la femme de chambre de la comtesse, Hauteclaire, portée disparue. La perplexité que cette découverte fait naître chez Tory est bientôt levée. Un concours de circonstances lui permet d'assister à une scène d'amour entre le comte et la domestique. Les deux complices jouent cependant sciemment les rôles qu'ils se sont arrogés et échappent à la suspicion de ceux qui les entourent. Seul le regard perçant du médecin examine minutieusement leurs faits et gestes. La comtesse s'affaiblit graduellement jusqu'à ce qu'un jour sa servante, s'étant trompée de fiole, lui fait prendre, au lieu du médicament prescrit, une encre double à base toxique. Appelé d'urgence, le docteur Tory recueille les dernières paroles de la comtesse de Savigny. C'est ainsi qu'il apprend que celle-ci, au courant de l'adultère de son mari, attribue son empoisonnement à un geste prémédité. Mais afin de ne pas souiller le titre noble associé à son nom, elle interdit au médecin de faire rayonner l'histoire du crime. Depuis, Hauteclaire et Serlon se marient et, au défi de la société, étalent au grand jour leur « étonnant et révoltant bonheur. » (120).

11 L'écriture du Bonheur souscrit pleinement à la technique narrative sur laquelle les commentateurs des Diaboliques ont peut-être le plus attiré l'attention: la mise en abyme des narrations, l'enchâssement du récit dans un récit initial qui lui sert de prétexte et de cadre. ${ }^{13}$ La succession de prise de parole, qui correspond à l'image figurée de Ricochets de conversations - titre provisoire que Barbey avait donné à son recueil en chantier accomplit deux choses à la fois : d'un côté elle authentifie l'histoire relatée (le narrateur n'invente pas; il ne fait que répéter ce que l'on lui a raconté), de l'autre, elle privilégie la dimension orale du récit (le narrateur premier, ayant déjà un interlocuteur à l'intérieur de la fiction, se constitue lui-même comme auditeur du second narrateur).

Il s'agit plus précisément d'une "soumission du récit à l'entretien", à «la causerie ", puisque ce "récit repose sur une situation orale ", pour reprendre les expressions de Michel Crouzet. "Venez par ici. Nous allons causer » (89), dit effectivement le docteur Tory au moment où il s'apprête à entamer son récit. Contre le culte du narrateur impersonnel et invisible auquel se vouent les romanciers réalistes et naturalistes, contre l'esthétique anonyme du "reportage », Barbey s'ingénie à singulariser son conteur, à le doter d'une physionomie et d'un tempérament, en même temps qu'il s'emploie à maintenir le contact avec le lecteur, à solliciter sa participation dans le processus de l'énonciation narrative. ${ }^{14}$

[C]'est une voix, un ton, un homme selon la vieille distinction classique de l'homme et de l'auteur, qui est présent devant le lecteur et présent dans sa parole. Il se communique par l'intermédiaire d'une mise en scène de lui-même au centre d'un auditoire fictif. On dira aussi qu'il doit ménager toujours une présence orale. De là 
ces transmissions de récits, ces courses de relais de narrateurs qui se passent la parole vivante, l'oralité narrative. ${ }^{15}$ aboutissant à une dilatation du régime énonciatif, en vient à relativiser la question des origines du récit et à brouiller celle de sa destination. ${ }^{16}$ Ce qui permet à Pierre Schneider de dire que "les événements nous arrivent dépersonnalisés par une succession de raconteurs qui se les sont transmis les uns aux autres. $\aleph^{17} \mathrm{Ne}$ faudrait-il pas voir dans cette "dépersonnalisation » des données narratives, résultant de la prolifération des énonciateurs, un premier indice de théâtralisation du récit? L'effacement du dramaturge, qui pour dire ne dispose que de l'élocution d'autrui, qui délègue sa parole pour ne s'exprimer que par des voix interposées, n'est-ce pas en fait ce par quoi d'abord l'énonciation théâtrale se définit comme telle?

rtant, si le premier narrateur du Bonheur s'efface, ce n'est pas pour disparaître de la diégèse. Il ne s'agit nullement ici d'un destinataire passif qui se contente d'être un simple réceptacle du discours de son remplaçant. Bien au contraire, il s'évertue à rappeler sa présence. Il supplie: «Docteur, mon cher et adorable docteur, - repris-je, avec toutes sortes de câlineries dans la voix» (88), questionne: «Et cela ne pouvait être que vous, docteur ?» (117), interrompt : «Hum! fis-je sceptiquement au docteur, que je ne pus m'empêcher d'interrompre » (104), commente : «Voilà qui est osé ! - dis-je au docteur » (101), relance : «Quoi, docteur, ils auraient donc?... (116), mais sait aussi rester discret dans l'intérêt de la narration: "Il me parut si intéressant à son tour, que je ne lui fis aucune observation et qu'il reprit.» (115). Non seulement il est irrémédiablement impliqué dans l'action de raconter, mais il en est profondément affecté : «[S]on histoire me pinçait. » (117). Le repli du premier « je » ne correspond donc en aucune manière à une retraite définitive du processus narratif, mais confirme a contrario la stratégie globale des Diaboliques qui structurent leur récits sur le principe du dialogue. Par là même Le Bonheur invite à une lecture adoptant la perspective propre à la dynamique théâtrale.

En effet, dès lors qu'une fiction narrative privilégie les dialogues sur les passages narratifs, la posture d'immersion de la narration naturelle a tendance à faire place à celle qui prévaut lors de la lecture d'une pièce de théâtre, c'est-à-dire que nous neutralisons provisoirement l'aspectualité narrative pour adopter la posture d'immersion qui correspond à celle de la fiction dramatique. ${ }^{18}$

L'économie compositionnelle du Bonheur qui relève du mode de la conversation et s'adosse sur la polyphonie narrative, justifierait ainsi à elle seule une approche analytique, conforme aux spécificités du texte dramatique. Mais le phénomène de la théâtralité tend à se manifester de façon plus patente encore. Les références lexicales, le recours à des images ou à des procédés traditionnellement associés au domaine du théâtre parsèment la narration.

Un trait caractéristique du conteur central est singulièrement mis en valeur dès les premières pages de la nouvelle, mais persiste infailliblement tout au long du récit: le premier narrateur qualifie le docteur Tory de "grand observateur (81) ou encore d'« impitoyable observateur $» \cdot{ }^{19}(83)$. Le docteur lui-même exalte « la pénétration de [s]on regard ». (123). C'est que raconter ici ne dépasse pas la reconstitution discursive de faits systématiquement observés. Chaque fois que le narrateur entreprend d'envahir le monde intérieur de ses personnages, chaque fois qu'il a recours à la logique pour comprendre les causes profondes des événements qu'il met en scène, son récit s'entrave et il se retrouve dans l'impossibilité de raconter. ${ }^{20}$ Tout au plus construit-il des hypothèses ou formule-t-il des questions auxquelles il ne peut répondre que par des suppositions.

Cahiers de Narratologie, 14 | 2008 
«Je commençai immédiatement de raisonner cette situation pour l'éclairer... Depuis combien de temps existait-elle?... Datait-elle de la disparition de Hauteclaire ?... Y avait-il déjà plus d'un an que la chose durait? [...] Toutes questions qui montèrent à cheval et s'en vinrent en croupe à V... avec moi, accompagnées de bien d'autres qui se levèrent et que je ramassai sur ma route. » (102).

17 Le narrateur est obligé donc, pour pouvoir raconter, de s'en tenir à ce qu'on pourrait appeler une esthétique de la vision. Dès lors, le thème du regard et ses dérivés se placent au centre du récit. Rien de surprenant ainsi à ce qu'en plus du don de l'observation exceptionnellement développé, le narrateur central soit également doté d'une parfaite vue, d'un « œil pénétrant, vierge de lunettes. » (82).

Sans doute, le jeu des regards, qui tentent de percer les surfaces et de démasquer les apparences, qui sondent et qui dissèquent, ${ }^{21}$ occupe une place privilégiée au cœur de l'univers diégétique. Le regard de Hauteclaire exerce le même pouvoir hypnotique sur les bêtes et sur les hommes. "[J]e vis ses yeux, à elle... ces yeux qui fascinaient des tigres. » (86). L'auteur lui-même s'attache à mettre en valeur, au sens fort du terme, l'importance de la fonction visuelle pour la logique du récit. «Il venait de voir que j'avais vu, mais il voyait aussi que je ne voulais rien voir de ce que j'avais vu et il respirait.» (102-3). Le regard s'applique à dominer une situation, à mettre l'autre à l'épreuve, mais peut aussi trahir et conduire ainsi au renversement du rapport de force entre le regardant et le regardé. Ayant retrouvé Hauteclaire sous l'habit de la femme de chambre, le docteur Tory croit à tort pouvoir soumettre la fausse servante à son autorité.

«[J]'eus cependant la petite faiblesse de vouloir lui montrer, à cette fille audacieuse, que je la reconnaissais. [...] je lui ai planté, à elle, mes deux yeux dans ses yeux, comme si j'ai enfoncé deux pattes-fiches ; mais ses yeux - de biche, pour la douceur, ce soir-là, - furent plus fermes que ceux de la panthère, qu'elle vient, il n'y a qu'un moment, de faire baisser. Elle ne sourcilla pas.» (102).

19 Mais si le docteur Tory s'abstient de dénoncer la supercherie, ce n'est pas parce qu'il est dompté par le regard fascinateur d'Eulalie ; c'est qu'il est curieux d'apprendre le fond de l'histoire, occultée par l'artifice d'une mise en scène élaborée. Le conteur du Bonheur, gouverné par une «curiosité acharnée » (103) - une "curiosité éternelle » (122) - sur laquelle le texte ne cesse de revenir, demeure fidèle à l'image générale de « chasseur aux histoires » (21), accordée aux personnages des Diaboliques, dès la première nouvelle. Et comment l'homme de science pourrait-il résister à cette chasse à l'histoire qui met à l'épreuve son esprit systématique? «Cela me prenait la pensée comme la griffe du sphinx d'un problème. » (110). Or, la solution de l'énigme ici est étroitement liée à la possibilité de voir. En s'interdisant de commettre une indiscrétion, le docteur Tory est à même de satisfaire "l'intérêt de l'observateur " et par là même les "plaisirs de l'observateur ", qu'il admet mettre "au-dessus de tous les autres». (103). Ce goût prononcé pour l'observation que cultive le narrateur se trouve rehaussé par une assimilation d'unités descriptives entières à des scènes de nature théâtrale.

De ces scènes, celle qui a été le plus fréquemment retenue par les exégètes du Bonheur décrit un Tory à qui le hasard offre l'occasion de contempler les ébats amoureux du couple adultère, mêlés à la pratique de l'escrime. C'est avec raison que cette scène a souvent été analysée dans une perspective plus ou moins psychanalytique. ${ }^{22}$ Le docteur s'adonne d'autant mieux aux délices du voyeurisme, que ses tentatives antérieures de percer le jeu des complices restent infructueuses. La stratégie du secret surpris, de l'intimité «violée » par un regard indiscret, est néanmoins un procédé dramatique par 
excellence. Pour peu que l'on s'attache à examiner la séquence narrative en question par le prisme de la théâtralité, on voit apparaitre sa dimension scénique. L'articulation de la description à un décor de théâtre est indéniable. Le guetteur tapi dans l'ombre du bois, à minuit, l'heure des mystères, le jeu de la lumière et de l'obscurité, la porte-fenêtre aux persiennes à moitié fermées, laissant deviner des mouvements dont on n'entend d'abord que le bruit ${ }^{23}$, le balcon où s'enlacent finalement les deux amants: tout semble concourir à produire l'impression d'une scène s'offrant au regard du spectateur. Et comme si cette prégnance dramatique n'était pas suffisamment convaincante, le narrateur s'attache à saturer son discours de termes à tonalité théâtrale. Les personnages observés " apparaissaient sur le fond lumineux qui les encadrait» avant de "s'accouder sur la rampe du balcon ». La porte vitrée éclairée " mettait en relief leur double silhouette », en même temps qu'elle permet de distinguer les détails de leur « costume ». Les paroles des amants sont étouffées, mais « les attitudes de leur corps les disaient pour eux ». Quand ils se retirent dans l'appartement, « ils abaissèrent les rideaux, de grands rideaux sombres ». (112-13). C'est un véritable inventaire théâtral que la narration met en place: la rampe, le rideau, le cadre, le costume, l'éclairage, le corps, toutes ces composantes créent sans équivoque l'illusion d'un espace scénique. On dirait ainsi avec Marcelle Marini que «le point focal du système narratif serait donc cette scène à deux acteurs surprise par un témoin qui à la fois en est exclu et s'y trouve impliqué. $»^{24}$

21 Mais si cette scène d'allure fondamentalement dramatique constitue le point tournant de la nouvelle, du point de vue sémantique aussi bien que topographique (elle se situe presque au milieu du récit proprement dit), elle est loin d'être le seul exemple où le narratif se pare ostensiblement d'attributs appartenant au théâtre. Comme si pour accentuer son importance décisive pour l'intrigue, deux autres épisodes à caractère théâtral; viennent l'encadrer. Le premier sert d'incipit au spectacle dont le château « était le silencieux et discret théatre " (106), dans la mesure où il inaugure l'apparition de Hauteclaire dans le costume de la servante Eulalie. Son entrée rappelle effectivement l'évolution sur scène d'une actrice, éclairée par le faisceau des projecteurs. «[J]e (Tory) vis émerger de l'ombre qui noyait le pourtour profond du parloir, et s'avancer au bord du cercle lumineux, tracé par la lampe autour du lit, Hauteclaire Stassin; oui, Hauteclaire elle-même ! [...] C'était à couper la respiration qu'une telle vue! Eulalie !...» (101-2). Le passage de l'ombre à la lumière a ici une valeur symbolique et symptomatique à la fois. Symbolique, car il se constitue comme une métaphore de l'illumination dont est frappé le docteur. Tiré de l'ombre de l'ignorance, celui-ci devient à partir de ce moment le détenteur d'un savoir que les autres ne possèdent pas. Symptomatique, parce que c'est encore par le biais de la vue que la révélation a lieu. Si cette scène fait pendant à celle examinée plus haut, c'est que là aussi l'acquisition du savoir est figurée à la faveur d'une transition de l'obscurité à la clarté, - une trajectoire qui part de "l'épaisseur du bois ", où on ne voit "goutte de lumière ", passe par l'image du "château blêmi par la lune " et aboutit à « la lumière d'un candélabre ». (110-11).

Le second épisode sert, à l'inverse, de clôture au projet théâtralisé mis à exécution par les deux complices. Il se produit au moment où éclate le scandale provoqué par l'annonce du mariage entre Hauteclaire et Serlon. "C'est dans le grand escalier du château que je (Tory) la rencontrai cette première fois. Elle le descendait et je le montais. Elle le descendait un peu vite; mais quand elle me vit, elle ralentit son mouvement, tenant sans doute à me montrer fastueusement son visage, et à me mettre au fond des yeux, ses yeux. » (124). L'emploi du verbe «montrer » résume à lui seul la dimension théâtrale de 
cet affrontement dépourvu de parole. Médiatisé une fois de plus par le regard, cet ultime défi vient couronner en quelque sorte un long combat dont Hauteclaire sort vainqueur. Sa position supérieure vis-à-vis de son adversaire, de même que «l'air heureux de la plus triomphante et despotique maîtresse » (124) attestent sa victoire qu'elle n'a pas besoin de dire puisqu'elle l'affiche sur son visage et en investit son regard. Vu sous cet angle, ce face à face se donne à lire comme le finale d'un spectacle domestique, joué dans l'intimité du " château de Savigny qui fut le théâtre d'un crime. » (127).

Du reste, il est impossible de ne pas tenir compte des indices de théâtralité dans un récit où l'histoire racontée est invariablement qualifiée de "comédie». En réalité toute l'originalité du projet concocté et mis en œuvre par le couple criminel réside dans son association à la représentation théâtrale. Or, c'est précisément ce passage obligé par la théâtralisation qui fournit quasi intégralement le matériau premier à la narration. Autant dire que celle-ci n'est possible ici que dans la mesure où elle procède à sa propre dramatisation. Le texte est particulièrement éloquent à cet égard. C'est par ailleurs la première impression que Tory enregistre après avoir reconnu l'escrimeuse disparue sous l'uniforme de la servante. "Ce qu'il y avait de certain, c'est que le comte de Savigny et Hauteclaire Stassin jouaient la plus effroyablement impudente des comédies avec la simplicité d'acteurs consommés, et qu'ils s'entendaient pour la jouer. » (104). À partir de ce moment, paradoxalement, l'acte de raconter (dans une œuvre de création) sera cautionné par l'existence d'une fiction et plus spécialement d'un spectacle, dont la raison d'être ne se laisse pas épingler. De l'observation à l'espionnage il n'y a qu'un pas, et Tory le franchit dans une tentative de prêter un sens cohérent aux faits observés: "Je mouchardais (oh! je ne m'épargne pas le mot) pour le compte de ma propre curiosité. » (110). Ce n'est pas pour autant que son savoir s'élargit. L'expérience de l'espion s'avère aussi décevante que celle de l'observateur. N'étant pas en mesure de fournir de nouvelle information et faire ainsi évoluer l'intrigue, le docteur, pour continuer de raconter, n'a d'autre alternative que de revenir sur le point de départ de son enquête: la certitude d'assister à un spectacle, conçu dans un dessein qui se dérobe à son raisonnement. Si astucieux que soit le conteur, il ne peut que s'en remettre aux apparences. Le discours narratif accomplit ainsi un parcours circulaire. «Savigny et Hauteclaire continuaient de jouer, avec la même perfection, la très difficile comédie que mon arrivée et ma présence au château n'avaient pas déconcertée. » (110). La révélation de la comtesse ne résout rien. La vérité profonde du comportement des deux amants qui « jouent toujours serré » (121) échappe à Tory. D'où une nouvelle série de questions stériles qui, s'étalant sur une demipage, camouflent mal l'échec de l'investigateur et par conséquent celui du narrateur. Le rôle central de la théâtralité, qui n'est pas démenti d'un bout à l'autre du récit, s'actualise de diverses manières et revêt différentes formes. Par exemple, le texte de la nouvelle n'hésite pas à faire appel à des personnages fictifs, appartenant à des œuvres théâtrales, pour décrire les postures et les attitudes de ses propres protagonistes. Le docteur Tory, qui est comparé à « un misanthrope à l'Alceste » (83), prend l'air « tragique comme la statue du Commandeur, quand elle accepte de souper. » (116). Hauteclaire, elle, ressemble à " une lady Macbeth, cette gaillarde-là.» (88). La lecture du "Mariage de Figaro ", suggère le narrateur, pourrait éveiller la méfiance de la comtesse vis-à-vis de cette « belle fille de chambre » (104).

Dans le même registre, le qualificatif d'«actrice » (97) que les habitants de la ville de V... accordent volontiers à l'escrimeuse est révélateur. "Admirée » de tous, suscitant des « curiosités ardentes » (93) et un « intérêt prodigieusement enflammé » (94), Hauteclaire 
finit par devenir «la grande artiste, la diva spéciale » (99) au sein de la communauté aristocratique. Dès lors que le docteur Tory surprend Hauteclaire à jouer le « rôle de servante » (106), il se sert principalement de l'expression «la fausse Eulalie » $(105,106$, $110,116,125)$ pour désigner son héroïne et ponctuer ainsi son don de l'incarnation. Mais si le narrateur se concentre sur la performance du personnage féminin, ce n'est pas pour autant qu'il se désintéresse du talent artistique du comte de Savigny. "Je me contentai de le regarder. Il devenait plus intéressant pour moi que jamais, car il m'était évident qu'il jouait plus que jamais la comédie.» (115). Il y a mieux : le narrateur lui-même est irréversiblement pris au jeu du simulacre. Simple spectateur au début, il se voit contraint, lui aussi, à incarner le rôle de « comédien » (114) afin de ne pas être écarté du château et privé, de ce fait, du théâtre qui lui apporte tant de plaisir.

Qui dit théâtre, dit déguisement. Il est donc logique que le masque, en tant qu'il est le symbole par excellence du déguisement, fasse partie intégrale du dispositif narratif du Bonheur. De ce point de vue, le choix opéré par Barbey quant à l'occupation de son héroïne semble n'avoir rien d'aléatoire. En fait, ce choix témoigne de deux implications importantes, éclairant notre propos. Il permet, en premier lieu, d'introduire dans la diégèse le concept de masque, ce qui autorisera le narrateur à faire une transition «naturelle » de l'univers de l'es-crime (où le masque protège) à celui du crime (où le masque dissimule); la seconde implication est une fonction de la première : l'imposition du masque et ses équivalents (voile, artifice vestimentaire, coiffe, etc.) au coeur du récit viennent confirmer la mobilisation du régime théâtral pour le compte de la narration. Les exemples à cet égard abondent ; contentons-nous des plus éloquents. ${ }^{25}$ " [L]e dimanche à la messe, comme dans la rue, elle était presque aussi masquée que dans la salle de son père, la dentelle de son voile noir étant encore plus sombre que les mailles de son masque de fer. » (94). S'exhiber ou se dissimuler devient ainsi une seule et même action pour le personnage de Hauteclaire. "Cette manière de se montrer ou de se cacher" (94) lui permettra plus tard de s'approprier un rôle justement en se découvrant. Les apparences prendront alors figure de réalité grâce à un simple dispositif vestimentaire. "Son déguisement [...] était complet. Elle portait le costume des grisettes de la ville de V..., et leur coiffe qui ressemblait à un casque. » (102).

Le thème du masque contient en puissance celui de l'identité. Si les voiles que Hauteclaire "épaississait à dessein " (104) ne rendent indistincts que les traits de son visage, son déguisement derrière l'uniforme de la femme de chambre tient lieu d'une véritable métamorphose. Se déguiser, ne revient-il pas au fond à emprunter une personnalité différente? Or, faire semblant d'être quelqu'un d'autre, c'est exactement ce qui caractérise le métier de comédien. Hauteclaire interprète son rôle " comme si elle avait été exclusivement élevée pour cela. » (105). Le narrateur ne se lasse pas de rappeler qu'au cœur de son récit se tient la fiction théâtrale, qu'on a affaire à un " comme si », que les fausses apparences se greffent sur la réalité et la déforment. Il ne semble pas se contenter de constater la singulière transformation que subit son personnage principal, mais tient en outre à scander sa duplicité. ${ }^{26}$ De là le double prénom qu'il attribue à son héroïne: « Hauteclaire-Eulalie ». (109).

C'est encore sur le plan de l'identité que l'acte théâtral, indispensable à l'accomplissement du crime, annonce sa fin. Le mariage conclu avec le comte de Savigny témoigne d'un ultime changement identitaire chez Hauteclaire, celui-ci non plus fictif, mais garanti au contraire par une institution officielle. «Lorsque je vis la fausse Eulalie parfaitement comtesse, elle me reçut comme si elle l'avait été toute sa vie.» (125). 
L'appropriation du titre de noblesse par la servante établit une réalité sociale qui contredit le système des valeurs établies. L'ironie aurevillyenne prend alors un ton particulièrement mordant. Devenue «authentiquement comtesse de Savigny » (124) c'est-à-dire précisément ce qui s'oppose à ses origines populaires ${ }^{27}$-, Hauteclaire n'a plus besoin de fuir le regard des autres. Dès lors que l'artifice l'emporte sur le réel auquel il se substitue, le masque n'a plus lieu d'être, le déguisement n'est plus de mise. « Maintenant, elle lève son voile et leur montre hardiment le visage de servante qui a su se faire épouser.» (127).

Ce n'est pas pour autant que la résonance théâtrale du récit se dissipe. Le verbe «montrer» prend ici (comme dans l'exemple relevé plus haut) une signification emblématique. Il témoigne d'une continuité et non d'une rupture. Le subterfuge, désormais inutile puisqu'il a rempli sa fonction, ne s'estompe que pour donner naissance à un nouveau type de théâtre, dont la spécificité consiste à faire valoir et à faire voir ce que justement son homologue précédent cherchait à disqualifier et à dissimuler. Si le couple continue de se "donner en spectacle », ce spectacle poursuit un but antithétique de celui réservé aux rares spectateurs du château: faire grand étalage d'un bonheur inconcevable et scandaleux. La scène, d'allure franchement exhibitionniste, jouée par ce couple altier « sous les exclamations du groupe populaire » (87), dans le Jardin des Plantes (lieu public par excellence qui fait contraste avec l'intimité du château), en est l'exemple le plus frappant. Cet épisode à charge dramatique, servant de prélude au récit du docteur Tory, se constitue aussi comme une grande anticipation qui résume son contenu et préfigure sa fin. Sous ce rapport, il n'est pas inessentiel que ce soit une référence à la spectacularité qui inaugure la narration et qui également la referme.

30 Si le récit donne à voir, grâce à sa théâtralité, plus qu'il ne révèle par l'enchaînement des événements relatés, c'est que la narration à elle seule est impuissante à rendre un compte exhaustif de la situation qui l'a engendrée. Manifestement, raconter ne suffit pas ici pour accéder à l'intériorité des personnages. Cette insuffisance narrative est soulignée sur plusieurs plans qui forment un réseau isotopique. Nous avons déjà insisté sur l'hermétisme caractéristique du personnage central. À l'impénétrabilité du masque et du voile qui dissuade le regard, se joint celle de la pensée qui désarme le psychologue. Aux yeux du docteur Tory, Hauteclaire semble « encore plus impénétrable de caractère que de visage. » (94). Inatteignable au sens figuré, elle l'est aussi au sens propre. « On ne peut pas vous toucher, Mademoiselle " (95), remarque paradoxalement, au cours d'une leçon d'escrime, celui qui ne tardera pas à devenir son amant avant d'être son mari. À l'inaccessibilité du corps, protégé par la « cuirasse » (113) du gilet d'armes, correspond la renommée immaculée. En dépit de la fréquentation d'une clientèle exclusivement masculine, «la réputation de Mlle Hautecalire ne fut [pas] effleurée.» (97). Qu'elle soit voilée ou non, qu'elle ait affaire à un de ses élèves ou à un observateur aussi ingénieux et expérimenté que Tory, ses pensées et ses sentiments demeurent indéchiffrables. «Sa physionomie [...] ne trahissait ni chagrin, ni préoccupation, ni rien de nature » (98) à suggérer le scandale à venir.

31 L'impénétrabilité tant physique que psychologique du personnage principal préside à la défaillance narrative dont témoigne le discours du docteur Tory. Bien que ce dernier finisse par être promu au rang de familier au sein de la vie conjugale - position lui permettant d'« étudier microscopiquement leur incroyable bonheur» (125) -, son enquête continue de piétiner. En vain s'acharne-t-il «à regarder et à percer dans l'intimité de ces deux êtres.» (127). Il en est amené à avouer l'inefficacité de son regard 
sur lequel s'appuie cependant la narration. «Je ne remarquai quoi que ce soit qui pût me renseigner sur le secret que j'avais surpris.» (105); ou bien: «Ce que je voyais ne me renseignait pas beaucoup. » (109); et encore : «Je ne voyais rien du roman qu'ils faisaient entre eux». (110). L'histoire du bonheur criminel qui cherche sa cohérence et son dénouement échappe au récit. La narration se désiste alors de l'anecdotique et s'oriente vers le tragique qui l'alimente et lui permet de se prolonger, « en attendant le drame et la catastrophe ». (110). Bref, elle se théâtralise conformément au matériau qui se tient à sa source. La dramatisation du discours narratif du Bonheur (le récit) s'assimile en fait à la nature théâtrale des événements (l'histoire) orchestrés par les deux complices. Ce qui représente emprunte la "recette» à ce qui est représenté. Car dite au moyen des procédés narratifs conventionnels, cette histoire compromettrait sa crédibilité et, par là même, la possibilité de sa réalisation. Simplement « racontée à n'importe qui, [elle] aurait semblé impossible. » (103). Pour être cru, il ne suffit pas seulement que le conteur offre à son auditeur un rapport détaillé des faits qu'il a observés ; il faut aussi que le récit ébranle son destinataire de la manière dont les événements qu'il retrace ont bouleversé le narrateur et l'ont incité à réviser son système de valeurs.

Dans la mesure où l'écriture de la nouvelle s'empare des moyens propres au genre dramatique pour s'actualiser, elle assume la fonction moralisatrice dont se réclament Les Diaboliques: mettre en scène des passions corruptrices dont le spectacle terrifiant conduirait à l'édification spirituelle du lecteur. "Je voulais parler aux âmes et non pas aux sens $\aleph^{28}$, insiste Barbey lors du procès des Diaboliques. On commence à comprendre mieux dès lors pourquoi la sensation prédominante que génère le récit du Bonheur est l'horreur. La communauté aristocratique apprend «avec terreur» (116) l'empoisonnement de la comtesse. Le monde a d'abord l'impression «d'un accident terrible" (116), mais bientôt le crime soupçonné fait naître un "horrible bourdonnement». (117). La mésalliance entre Serlon et Hauteclaire est qualifiée de «monstrueuse » (116), le scandale déclenché par leur mariage d'«épouvantable » (122), les deux personnages eux-mêmes sont appelés des «monstres» (125); la comtesses Savigny, sur son lit de mort est "effrayante » (117), sa confidence, quant à elle, est «terrible ». (123). Mais plus révélatrice encore est la réaction de celui qui, récepteur du récit au cœur de la fiction, incarne de toute évidence la figure du lecteur impliqué. ${ }^{29}$ "J'étais épouvanté... “Mais, - fis-je, si c'est vrai ce que vous me contez là, docteur, c'est un effroyable désordre dans la création que le bonheur de ces gens-là."» (120). Envisagé par le prisme de la formule aurevillyenne citée plus haut - «moraliser par la terreur » - cette prolifération démesurée, dans les dernières pages de la nouvelle, de termes apparentés auxquels l'horreur sert de dénominateur commun prend un sens plein. La narration cherche à agir sur le lecteur de manière analogue à celle qui est à l'œuvre dans le genre tragique. La stratégie narrative consiste à engendrer, par le truchement de la frayeur, des effets cathartiques, aptes à associer le plaisir esthétique de la lecture à une expérience de purification morale. Tel semble être le but recherché par la narration d'inspiration théâtrale du Bonheur et plus généralement par celle de l'ensemble des Diaboliques. Ainsi pourrait s'expliquer le phénomène selon lequel les textes des différentes nouvelles débouchent invariablement sur l'horreur et la fascination, le malaise et la rêverie d'un auditoire pris à parti et soumis à la puissance transformatrice d'un récit, à saturation dramatique plus ou moins dense..$^{30}$

On constate, cependant, que la stratégie déployée est loin de se conformer à la poétique de la tragédie classique. À regarder de plus près, en effet, le modèle tragique d'épuration 
morale semble être compromis sur deux points. La fin du récit insiste (et c'est précisément en cela que consiste le diabolisme de la nouvelle) sur un manque flagrant de punition. Le fatum, élément emblématique du genre tragique, brille ici par son absence. D'autre part, le bonheur insondable, et par là même fascinant, n'est pas en mesure de déclencher le processus cathartique (la purification ne peut pas s'alimenter de ce qui est inintelligible). À supposer même que Barbey ait trouvé un nouveau régime de l'horreur, celui-ci ne fonctionne pas sur le mode tragique, mais se négocie, à l'opposé, au nom d'une immoralité scandaleuse. La seule manière de comprendre ici ce «bonheur dans le crime ", c'est de l'admettre et de le partager, d'en devenir complice, d'en répandre l'histoire. Autant dire de bloquer toute possibilité de catharsis, au sens canonique du terme. Il faut donc remettre en perspective le statut du discours préventif de la seconde préface des Diaboliques dans sa référence plus ou moins nette à l'art de la tragédie et à ses enjeux moraux ; car ces derniers deviennent paradoxalement « victimes » des prétentions moralisatrices d'une théâtralité qui éloigne le lecteur de la purgation tragique plutôt que de l'en rapprocher.

Entre la première et la dernière Diabolique, entre Le Rideau cramoisi où justement "le double rideau » (21) - formant un "carré vide, rouge et lumineux» (57) qui rappelle étrangement celui de la scène théâtrale ${ }^{31}$ - ne se lève jamais, en sorte que le récit une fois achevé laisse les interlocuteurs contemplatifs dans l'obscurité et le silence de la diligence - métaphore incontestable de l'incompréhension, mais symbole aussi de l'espace physique de la salle de spectacles - et La Vengeance d'une femme, où l'enquêteur, mystifié d'abord par la forte théâtralisation d'une vengeance inédite, finit par tout savoir car, ici, précisément, la narration se pose comme but de faire « jaillir une hideuse clarté » (253) « Il commençait alors de comprendre - le rideau se tirait !» (252) -, il y a la théâtralité du Bonheur qui fait voir et qui suggère sans prétendre tout débrouiller, qui s'ingénie à montrer mais se refuse à conclure. ${ }^{32} \mathrm{En}$ ce sens, il n'est pas étonnant que ce soit dans l'ambiguïté, sur un mode hypothétique et par un "peut-être" nostalgique que s'achève cette nouvelle, oscillant entre la logique formelle du récit et l'économie esthétique de la tragédie, ${ }^{33}$ entre le narratif et le dramatique: " "Sans son crime, je comprendrais l'amour de Serlon". "- Et peut-être même avec son crime !" - dit le docteur. "Et moi aussi" - ajouta-t-il. » (128)

Prise au sens large, la logique structurelle de cette nouvelle met en cause les postulats de l'écriture réaliste. Le récit du Bonheur, à grand renfort de figures théâtrales, problématise la validité de la narration impersonnelle et omnisciente, telle qu'elle est pratiquée par les adeptes du réalisme et du naturalisme. ${ }^{34} \mathrm{En}$ multipliant les échecs et les impasses auxquels aboutit le récit, en préférant l'énonciation à l'énoncé, le particulier à l'anonyme, en substituant le masque à la révélation, l'opacité à la transparence, c'est au régime narratif réaliste que s'en prend Barbey. En effet, le discours du docteur Tory, qui fonde sa vérité sur l'observation et la surveillance, souscrit clairement à l'arrogance réaliste. ${ }^{35} \mathrm{Or}$, cette prétention à l'exhaustivité de l'expérience, à l'exactitude des descriptions et au déterminisme scientifique finit par subir une éclatante défaite que le conteur est le premier à admettre. Et puisque "ce bonheur [est] incompréhensible", en est amené à constater Tory, «il n'a plus de description ». (126). Aussi éloquent que soit le narrateur, son récit demeure inapte à établir un rapport de causalité probant entre les faits qu'il rapporte et la réalité, dont ces mêmes faits sont censés représenter l'image exhaustive. Indescriptible, la vérité se dérobe à l'acte de raconter; inflationniste, la narration ne parvient pas à garantir la production de sens. Le conteur finit ainsi par nier 
l'autosuffisance de son propre récit, dont la justification, en dernière analyse, ne consiste qu'à peindre « le spectacle auquel [il] assiste depuis tant d'années » (126) et la fascination toujours plus puissante qui en émane.

\section{NOTES}

1. Jules Barbey d'Aurevilly, Les Diaboliques (texte commenté et présenté par Michel Crouzet), Paris, Imprimerie Nationale, 1989, p. 352. Voilà ce qui pourrait expliquer aussi en partie pourquoi Barbey, qui n'ignorait rien de la prédisposition hostile de la critique à son égard - pour avoir pleinement contribué à l'attirer -, décide de reprendre les points essentiels de sa préface (en particuliers ceux concernant la moralité de la littérature) dans une sorte d'avertissement au lecteur, qu'il place, de manière incongrue, en tête de la dernière Diabolique, La Vengeance d'une femme.

2. Jules Barbey d'Aurevilly, Cuvres romanesques complètes (textes présentés, établis et annotés par Jacques Petit), Paris, Gallimard, coll. « Bibliothèque de la Pléiade », 1966, t. II, p.1293. Désormais, toutes les citations non identifiées en notes renvoient à cette édition.

3. Michel Crouzet, Les Diaboliques, ouvr. cité., p. 352.

4. Andrée Hirschi, «Le 'Procès' des Diaboliques », in Barbey d'Aurevilly 9 : l'histoire des Diaboliques (textes réunis par Jacques Petit), Paris, Lettres Modernes, Minard, 1974, pp. 5-64, 17-21.

5. Cette formulation n'est pas sans rappeler celle de Barthes, qui définit la théâtralité comme l'échange simultané et discontinu de plusieurs signes (paroles, gestes, etc.) : « une véritable polyphonie informationnelle, [...] une épaisseur de signes. » Roland Barthes, «Littérature et signification » in Essais Critiques, Paris, Éditions du Seuil, coll. « Tel Quel », 1964, p. 258.

6. Cité d'après Philippe Berthier, «Les Diaboliques et la critique française », in Barbey d'Aurevilly 9 : l'histoire des Diaboliques, ouvr. cité., 65-106, p. 88.

7. Dans l'intérêt de la perspective adoptée ici, il convient d'attirer brièvement l'attention sur le salon où prend lieu la narration du Plus Bel amour de Don Juan, ainsi que sur la disposition et les postures des personnages de cette nouvelle. Littéralement sous nos yeux, ce salon se transforme en une véritable salle de spectacle, tandis que les personnages assument les rôles respectifs de comédien et de spectatrices. La dynamique théâtrale est ici incontestable. Tel un acteur sur scène, le conteur est placé au milieu de «ce cercle rayonnant de douze femmes » (63) «Il étreignit d'un regard circulaire toutes ces femmes qui formaient autour de la table une si magnifique ceinture.» (66). « Elles se fondaient d'attention, en le regardant. Elles le buvaient et le mangeaient des yeux » (67). 8. Françoise Gaillard, dans son article « La représentation comme mise en scène du voyeurisme » (Revue des Sciences Humaines, 154 (1974 : Avril-Juin), pp. 267-82), dont l'analyse assimile le phénomène du voyeurisme dans cette nouvelle de Barbey à un effet d'illusion réaliste, remarque avec raison que la narration crée « un espace coincé entre les exigences de visualisation calqués sur la représentation dramatique, et celles de l'information brute du discours. » p. 269.

9. Daniel Grojnowski, Lire la Nouvelle, Paris, Dunod, 1993, p. 38. 
10. Ibid., pp. 37-38.

11. Ibid., p. 77.

12. À ces deux manifestations de la théâtralité, on peut ajouter le traitement du temps au cœur de la nouvelle. L'étude de Raymonde Debray-Genette, dont une partie est consacrée à l'analyse de la temporalité dans le Bonheur, soutient que le temps de la parole est le moment essentiel de la narration. Par conséquent, les autres niveaux temporels lui sont subordonnés en tout point. La réminiscence ne cherche pas alors à faire resurgir le passé d'un souvenir « mais à activer le présent sous forme de mise en scène avec perspective d'éloignement. Ce conte n'est pas une évocation du temps passé, mais une vision quasi immédiate, le temps de conter. " «Un récit autologique : Le Bonheur dans le crime ", Romanic Review, 64:1 (1973 : Jan.), pp. 38-53, p.43. M. Crouzet fait écho à ce commentaire et le complète : « La nouvelle parlée, et parlée d'une manière personnelle, actuelle, vivante, de manière à secouer les conventions romanesques, livre l'histoire au jeu du dialogue. » Loc. cit., p. 17.

13. Debray-Genette, par exemple, parle de « dénivellations narratives » ou d'une « narration en paliers » pour décrire le phénomène de la délégation narrative dans cette nouvelle (op. cit., p. 39 et p. 42). La mise en oeuvre de cette technique dans le Bonheur n'a pas cependant la complexité qu'illustre Le Plus bel amour de Don Juan, où on peut discerner trois niveaux d'enchâssement. Voir à ce sujet l'analyse de Marie-Claire RoparsWuilleumier (« Le Plus bel amour de Don Juan : narration et signification », Littérature (1973 : Février) 118-125), qui remarque qu'à la triple mise en abyme du narrateur se superpose l'emboîtement de trois lieux de récit, trois salons. (pp. 122-123).

14. Anne Giard, parmi d'autres, voit dans l'inachèvement de l'histoire - procédé narratif caractéristique de tous les récits des Diaboliques - une invitation, voire une exigence adressée au lecteur à qui incombe la tâche de combler les blancs du texte troué.

«L'auteur a une conception très moderne du lecteur, dont il exige un travail. » « Le Récit lacunaire dans Les Diaboliques », Poétique, 11 (1980), 39-50, p. 49.

15. M. Crouzet, loc. cit., p.13.

16. Rappelons à ce sujet que le vrai récepteur de l'histoire n'est pas le premier « je » mais un « madame » anonyme qui, à peine mentionné dans les pages d'ouverture de la nouvelle, disparaît énigmatiquement pour ne plus refaire surface.

17. Pierre Schneider, « Barbey d'Aurevilly l'extrême », Les Temps modernes 65 (1951:Mars), 1542-60, p. 1548.

18. Jean-Marie Schaeffer, Pourquoi la fiction? Paris, Éditions du Seuil, 1999, pp. 278-9.

19. Cette information constitue ce que Debray-Genette a appelé ailleurs " une amorce ». Son importance pour le dispositif narratif et sa véritable portée ne nous est pas révélée immédiatement mais au fur et à mesure que progresse l'histoire. « Les figures du récit dans 'Un Cœur simple' », Poétique 3 (1970), pp. 348-364.

20. Dans cette transmission d'un savoir fragmentaire qui ne fait que refléter la surface des choses, Anne Giard voit la manifestation d'un refus des techniques romanesques conventionnelles. « Le narrateur en est réduit à décrire des comportements dont il souligne l'étrangeté, parce qu'il ne peut pas (et sans doute parce que Barbey d'Aurevilly ne veut pas) pénétrer dans l'intimité d'une investigation psychologique traditionnelle. » (A. Giard, loc. cit., p. 45).

21. Le personnage du docteur Tory fait montre d'une prédilection pour les métaphores médicales (celle de la sonde surtout, mais aussi celle de la dissection), pour décrire l'examen méticuleux, touchant au voyeurisme, auquel il soumet la vie intime du couple. 
22. Pour F. Gaillard, par exemple, la narration du Bonheur constitue une « figuration du fantasme » et met en scène un « transfert fantasmatique " puisque « raconter, c'est entraîner l'autre dans la situation de voyeurisme qui fut celle du narrateur. » Loc. cit. p.

267.

23. Ce bruit, dont la sonorité claire rend presque palpable est, lui aussi, doté d'une dimension visuelle : « On entendaient les épées comme si on les avait vues. » (112).

24. Marcelle Marini, « Ricochets de lecture: la fantasmatique des Diaboliques », Littérature (1973 : mai), 3-19, p. 11.

25. Dans la mesure où la figure du masque instaure l'opacité comme une nécessité narrative, une condition dont l'acte de raconter a besoin pour se réaliser, sa thématisation ici s'inscrit dans un registre plus ample de contestation de l'écriture réaliste (on y revient) qui, elle, se réclame de la visibilité du réel autant que de la transparence du discours qui le représente.

26. Il convient de noter au passage qu'à l'identité versatile de Hauteclaire s'oppose celle inaltérable de la comtesse. À cet égard, la scène du miroir (107) qui ne renvoie à celle-ci (et au lecteur) que les marques distinctives de sa propre individualité, prend un sens métaphorique. Elle atteste de manière suggestive l'existence d'une identité immuable chez le personnage noble. Écrivain marqué par une profonde nostalgie de l'Ancien Régime, Barbey saisit toute occasion pour dénoncer les « plates mœurs modernes » (105). L'antithèse entre les deux personnages féminins, indiquée par les origines modestes de Hauteclaire auxquelles font contraste celles, aristocratiques, de la comtesse, se trouve reproduite sur le plan plus subtil de l'identité, multiple et mobile pour la fille du peuple, unie et permanente pour la représentante de la noblesse. Précisons cependant que Barbey s'attache en même temps à brouiller la frontière de la distinction sociale, trop nette selon son goût prononcé pour la duplicité: Hauteclaire pratique l'art de l'escrime, symbole aristocratique par excellence ; son prénom aussi, à la différence de son nom, a des connotations nobiliaires et légendaires.

27. C'est l'Histoire qui s'empare de la narration ici. Le sort réservé à la comtesse de Sévigny et l'ascension sociale de Hauteclaire rejoignent les bouleversements historiquement datés, qui marquent le déclin de l'aristocratie et la restructuration de la société française. Helmut Schwartz remarque en ce sens : « Le crime de Serlon et de sa maîtresse est [...] un crime non seulement contre un individu, mais aussi contre une classe sociale. » Cité d'après Hermann Hofer, « Barbey parmi les Allemands », in Barbey d'Aurevilly 9 : l'histoire des Diaboliques, ouvr. cité., 121-43, p. 132.

28. Andrée Hirschi, loc. cit., p.18

29. «Barbey does choose his readers outside the text, but brings them into the fiction in the form of his narrator-turned-listener/reader ", remarque en ce sens Eileen Sivert, Symposium 31 (1977), 151-164, p.159. M. Crouzet, pour sa part, rappelle : « L'auditoire des Diaboliques (qui est nous-mêmes, lecteurs) est partie prenante dans le récit. » Loc. cit., p. 17.

30. Anne Giard établit un principe d'équivalence entre la réaction des auditeurs fictifs des Diaboliques et « l'effet que Barbey d'Aurevilly veut produire sur ses lecteurs réels, puisque cette réaction représentée doit non seulement refléter la leur, mais l'intensifier en ricochant sur elle. » Loc. cit., p. 49.

31. On rejoint ici le commentaire d'Arielle Meyer : « Il y a là, à n'en pas douter, un motif théâtral qui fait de ce récit non seulement une parole que l'on écoute mais, par l'intermédiaire de ce cadre éclairé et protégé par la pourpre d'un rideau, une scène que 
l'on regarde avec les yeux de l'imagination. » Le Spectacle du secret : Marivaux, Gautier, Barbey d'Aurevilly, Stendhal et Zola. Genève, Librairie Droz, 2003, p. 184.

32. Dans le Bonheur, effectivement, le narrateur ne comprend jamais qu'à moitié et il est le premier à le reconnaître. « Il y avait là un dessous que je ne comprenais pas. » (121). Sans doute peut-on voir ici un clin d'œil fait à la Diabolique suivante, Le Dessous de cartes d'une partie de Whist, où le jeu de cartes s'articule à celui de théâtre, dans la mesure où les personnages-joueurs s'évertuent à jouer "à la société une comédie dont elle est dupe. » (155). Mais cette phrase renvoie aussi, bien que de manière oblique, à la première nouvelle, Le Rideau cramoisi, où le personnage central féminin, pareil à Hauteclaire, fait preuve d'une audace extraordinaire. Rappelons sans insister que l'héroïne, qui incarne la duplicité au plus haut degré, ose flirter avec son amant secret au-dessous de la table et sous les yeux de ses parents, «qui ne se doutaient pas, dans leur placidité, du drame mystérieux et terrible qui se jouait alors sous la table. » (34).

33. Selon Dominique Rabaté, de manière générale, la tendance du tragique à se glisser dans le narratif relève du mouvement de la modernité en littérature. Privée de forme pour s'actualiser à partir de l'époque romantique, la tragédie, soutient le critique, « commence à se répandre, s'infuser et se diluer partout. [...] le tragique lui se glisse dans toutes les formes disponibles. Il contamine tous les genres; il devient indissociable de toute mise en forme, au point de devenir un composant essentiel de tout récit. » Poétiques de la voix, Paris, Librairie José Corti, 1999, p. 211. On voit qu'une telle contamination ne va pas sans trahison.

34. C'est tout le contraire de l'image du romancier réaliste qui se dégage de l'écriture aurévilyenne, dans la mesure où les péripéties, les hésitations et les carences de la narration personnelle l'emportent sur l'intérêt de l'intrigue. « Plus que tout autre conteur du dix-neuvième siècle, écrit Timothy Unwin, Barbey nous laisse entrevoir l'artiste au travail, qui essaie de faire surgir une aventure de l'oubli, et dont le sujet est justement cet effort de ressuscitation. " « Barbey d'Aurevilly conteur : discours et narrations dans Les Diaboliques », Neophilologus 72:3 (1988:July) 353-365, p 363.

35. Pur produit de la science moderne, soumettant le réel au calcul mathématique « cette inconnue de mon algèbre, je tenais à la dégager (109) -, le docteur Tory est aussi anticlérical. Son cynisme stigmatise le « catholicisme dont il était ennemi. » (89). Clairement, le personnage du médecin incarne les tendances positivistes qui gouvernent les rapports sociaux tout au long du XIX ${ }^{\mathrm{e}}$ siècle. Or, ce représentant des temps modernes, qui met « ses deux yeux aussi avant » (126) qu'il peut dans ce qu'il examine et qui en tire " en médecin des conséquences ", devient paradoxalement la victime de cette même transparence et de cette même logique, auxquelles il confie entièrement le succès de son entreprise, puisqu'elles finissent par « tromper [s]es prévisions. » (113).

\section{RÉSUMÉS}

C'est de l'imbrication du narratif et du théâtral, de la cohabitation entre récit et spectacle, entre « raconter » et « voir » que cet article, prenant pour exemple Le Bonheur dans le crime de Barbey d'Aurevilly, se propose de rendre compte. Ce qui donne un statut particulier à la troisième 
Diabolique dans l'optique de la théâtralité, c'est que l'intrigue repose ici entièrement sur le jeu de théâtre auquel se prêtent les personnages et sans lequel, au fond, le récit n'existerait pas. En fait, toute l'originalité de l'histoire réside dans son association à la représentation théâtrale. Il s'ensuit que l'acte de raconter (dans une œuvre de création) se trouve garanti par l'existence d'une fiction et plus spécialement d'un spectacle. Autant dire que le récit n'est possible ici que dans la mesure où il procède à sa propre dramatisation : la mise en intrigue s'assimile à la nature théâtrale des faits rapportés; ce qui représente emprunte sa stratégie à ce qui est représenté. L'article s'attache à examiner le rapport singulier qu'entretiennent narration et théatre et vise à dégager les implications qui en résultent. Si le récit de la nouvelle donne à voir, grâce à sa théâtralité, plus qu'il ne révèle par l'enchaînement des événements, c'est que la narration semble se donner comme objectif de dénoncer l'impuissance de l'écriture du roman réaliste, au profit d'une prétention à l'exemplarité moralisatrice, conformément à la poétique de la tragédie. Cependant, l'épuration cathartique par la lecture, énoncée dès la préface des Diaboliques et suggérée par la théâtralisation intensive du récit, ne se trouve-t-elle pas démentie par un dénouement qui semble saboter les principes mêmes du régime tragique?

Taking as its example Le Bonheur dans le crime by Barbey d'Aurevilly, this article proposes to discuss the interconnectedness of narrative and theater, the cohabitation of story and performance, and the relationship between "telling" and "seeing". What lends the third Diabolique its importance in terms of theatricality is the reliance of its plot on the interplay of theater, without which, in the final analysis, the narrative would not exist. Indeed, the originality of the story resides in its association to a theatrical representation. It follows that, in a fictional work, the act of narration is guaranteed by virtue of the existence of fiction. In other words, the story is possible only in so far as it dramatizes itself. This means that what the narrative represents draws its strategy from what is represented. The article will examine the peculiar relationship established between narration and theater and will attempt to shed light on the resulting implications of this relationship. If, thanks to its theatrical nature, the novella's plot hints at more than it presumes to reveal through the succession of its events, it is that the narration appears to adopt as its objective the denunciation of realist fiction as ineffectual, while upholding the exemplary moral nature to which conforms the poetics of tragedy. Yet, does not the cathartic release through reading, as enunciated in the preface of Diaboliques and suggested by the intense theatralization of the story, find itself contradicted by a denouement that outwardly sabotages the very principles of tragedy?

\section{AUTEUR}

\section{KRIS VASSILEV}

Associate Professor,Languages and Cultures, William Paterson University, New Jersey, États-Unis. 